\title{
Fish are Sensitive to Expansion-Contraction Color Effects
}

\author{
Liliana Albertazzi $^{1 *}$, Orsola Rosa-Salva ${ }^{1}$, Osvaldo Da Pos $^{2}$, and Valeria Anna Sovrano ${ }^{*}$ \\ ${ }^{1}$ University of Trento, Italy \\ ${ }^{2}$ University of Padova, Italy \\ *Corresponding authors (Email: liliana.albertazzi@unitn.it or valeriaanna.sovrano@unitn.it)
}

Citation - Albertazzi, L., Rosa-Salva, O., Da Pos, O., Sovrano, V. A. (2015). Fish are sensitive to expansioncontraction color effects. Animal Behavior and Cognition, 4(3), 349-364. https://doi.org/10.26451/abc.04.03.12.2017

\begin{abstract}
Some visual phenomena reveal the visual system's tendency to structure the scene according to rules that do not always have a direct correspondence in the external physical world. Color information can affect the perception of other object attributes such as size, generating contextual expansion, and contraction effects. We investigated phenomena of this kind in redtail splitfin fish (Xenotoca eiseni, family Goodeidae). As in previous studies, the fish were trained to discriminate a larger from a smaller grey rectangle in order to locate the exit from the test tank. The fish were then presented with two rectangles of equal size but different colors. Isoluminant colors were employed for each pair to control for luminance contrast from the background. The fish were tested in three different conditions with three color pairs corresponding to the human yellow-blue, red-green, and red-blue. The three color test conditions affected fish responses in significantly different ways. In the blue-yellow condition the fish seemed to experience the expansion-contraction effects as reported by human observers. By contrast, no difference between the choices of the two training groups was observed in the red-green and red-blue conditions. This is the first demonstration of human-like phenomenal chromatic expansion-contraction effects in non-human animals. Notably, the effect of the yellow-blue pair, which is usually the one most pronounced in humans, seems to be robust also under conditions of isoluminance in our model species. Future studies could repeat the experiment using non-isoluminant pairs that better preserve the connotative chromatic dimensions in humans.
\end{abstract}

Keywords - Psychological color dimensions, Contextual phenomena, Figure/ground organization, Redtail splitfin (Xenotoca eiseni), Fish, Color perception

An interesting aspect of animal cognition is the presence of similarities in perceiving the surrounding environment between humans and several other species of animals, despite their considerable ecological and genetic distance. In particular, fish species, given their great taxonomic diversity and phylogenetic distance from other vertebrates (Kumar \& Hedges, 1998), may furnish significant insights into the possible evolution of the vertebrate visual system, providing information on the neural correlates of perceptual organization in species belonging to different Taxa. It is striking, for example, how various species behave in response to a few contextual phenomena, as perceived by the human visual system (Coren \& Gyrus, 1978; Gregory, 1966; Oyama, 1960; Wade, 2005, 2010). At present, there is no general agreement on the nature and the role of so-called perceptual illusions in humans (Callander, Jennions, \& Backwell, 2011; Henrich, 2008; Henrich, Heine, \& Norenzayan, 2010; Segall, Campbell, \& Herskovits, 1963), or other animals (Kelley \& Kelley, 2014; Reaney, 2009; Wyzisk \& Neumeyer, 2007). However, the pervasive presence of these phenomena in different species sheds light on the principles that integrate the visual sensory stimulations into a coherent whole (Mascalzoni \& Regolin, 2011; Nieder, 2002; RosaSalva, Rugani, Cavazzana, Regolin, \& Vallortigara, 2013; Vallortigara, 2006, 2009, 2012; Vallortigara, 
Chiandetti, Rugani, Sovrano, \& Regolin, 2010), and it suggests that the Gestalt laws of organization could be common to several species belonging to different vertebrates' classes (Sutherland, 1961). Inhabiting the same physical world, simple organisms also have to be equipped with mechanisms to extract biologically meaningful units: the objects. For a similar reason, the Gestalt laws of perceptual organization may govern several aspects of animal behavior. Among fish, it has been shown that redtail splitfins (Xenotoca eiseni) prefer to process hierarchical stimuli at the global rather than local level (Truppa, Sovrano, Spinozzi, \& Bisazza, 2010), suggesting remarkable similarities between their visual system and that of other vertebrates, and prompting the hypothesis that fish discriminate stimuli according to a kind of perceptual categorization analogous to that of humans.

Teleost fish are able to discriminate two- and three-dimensional shapes (for a review: Agrillo, Miletto Petrazzini, \& Dadda, 2013; Rosa Salva, Sovrano, \& Vallortigara, 2014; see also Fuss, Bleckmann, \& Schluessell, 2013, for elasmoblanchs) and it has been shown that several species have also excellent trichromatic color vision (Beauchamp, 1978; Oyama \& Jitsumori, 1973), and are sensitive to color contrast effects (Burkamp, 1923; Dörr \& Neumeyer, 1996, 1997; Herter, 1950), brightness illusions (Agrillo, Miletto Petrazzini, \& Bisazza, 2016), and motion illusions (Gori, Agrillo, Dadda, \& Bisazza, 2014). Fish have also shown the capacity to discriminate illusory shapes (Wyzisk, 2005; Wyzisk \& Neumeyer, 2007; see also Rosa Salva et al., 2014). The tendency of redtail splitfins (Xenotoca eiseni, family Goodeidae) to perceive contextual phenomena affecting the metric size of the object in a similar manner to humans has been tested for the Ebbinghaus illusion (Sovrano, Albertazzi, \& Rosa Salva, 2015) and the Müller-Lyer illusion (Sovrano, Da Pos, \& Albertazzi, 2016). To investigate the perception of the Müller-Lyer and Ebbinghaus illusion, the fish were trained to discriminate between two stimuli of different sizes (two lines of different lengths for the Müller-Lyer illusion or two grey disks of different diameters for the Ebbinghaus illusion). After reaching the learning criterion, the fish performed the test trials, in which they were presented with two stimuli (lines or disks) of identical size. The two stimuli differed only in the context (i.e., the inducers). For the Müller-Lyer illusion, the inducers were two segments at the ends of each line oriented either inward, outward, or perpendicular to it. For the Ebbinghaus illusion, they were a circle of orange disks surrounding the target grey disk, which could be wider or smaller than the grey disk. The fish chose the figure that appeared either longer/wider or smaller/shorter, in accordance with the prediction of the illusions, and consistently with the condition of the training (Sovrano et al., 2015, 2016). However, less attention has been paid to the role of color dimensions that might be responsible for similar perceptual effects.

The conditions inducing the contraction/expansion effect in perceived visual surfaces have been shown through experimental and Gestalt analyses, although the research has mainly concerned the field of achromatics. For example, studies have been conducted on the contraction-expansion due to the segmentation of an object, such as a line or a long rectangle, as in the Kiesow effect (Kiesow, 1906); the apparent expansion of textured surfaces as in the so-called Oppel-Kundt illusion (Bazzeo \& Zanuttini, 1981; Oppel, 1860); the apparent shrinkage or expansion of striped surfaces (Bazzeo \& Zanforlin, 1984); the phenomenal shrinkage of a partly occluded figure (Kanizsa, 1972), and the phenomenal expansions of its modally visible parts (Kanizsa \& Luccio, 1978), attributable to amodal completion.

In the field of the arts, Itten (1961) showed that a white square on a black background appears to be larger than an identical square on a white background, because the white irradiates and expands the limits of the square, while the black contracts them. Less extensive have been the studies of the effect in the chromatic field that show the role of color as a gestalt-forming factor. Color gestalt is the set of colors that is perceived within an area. A color element is denoted by the particular object it imbues and by the color that fills it in (Hård \& Sivik, 2001). Descriptions of color dimensions influencing size perception have been developed in the field of the arts: color can modify shape appearance and its location in visual or pictorial space. However, one should bear in mind that Hering's (1964) theory of color is based on a formal analysis of color similarity: color interactions can be chiefly described by their mutual similarity. Various interactive connotative properties contribute to the phenomenon of contraction/expansion of surfaces with chromatic colors, such as the contrast between the polarities of warm and cold, light and dark, and close and distant (Albers, 1975; Katz, 1935). Put briefly, the warm colors are lighter and 
perceived as larger and closer to the observer; the cold colors are darker and perceived as smaller and more distant in space. The difference increases with very light and very dark colors, such as yellow and blue on warm and cold colors (see Da Pos \& Valenti, 2007). Schopenhauer (1994), studying the lightness relationships among complementary colors, found that yellow is three times lighter than violet, which means that a violet surface must be increased three times in comparison with a yellow surface to equilibrate the couple (see also Klee, 1961). Itten (1961) has maintained that the harmonic balance between colors (yellow, orange, red, purple, blue and green) is related to their area sizes. Kandinsky has described the centrifugal/centripetal effect of warm and cold colors in expanding/contracting (and spacing in depth) the visual surface perceived. For example, yellow is perceived to expand centrifugally and is perceived as closer, and blue shows the opposite effect (Kandinsky, 2001). In short, colors have apparent size, and yellow is perceived as the largest of colors, followed by white, red, green, blue, and black. In experimental psychology, research has shown the influence of hue and lightness on evaluation of perceived size. For example, it has been shown that colored areas have an influence on size perception (Claessen, Overbeeke, \& Smets, 1995; Cleveland \& McGill, 1983; Gentilucci, Benuzzi, Bertolani, \& Gangitano, 2001; Gundlach \& Macoubrey, 1931; Payne, 1964; Tedford, Bergquist, \& Flynn, 1977; Wallis, 1935; Warden \& Flynn, 1926) in various contexts, including pictorial space. These studies have been conducted with different objects (paper cards, slide projectors, colored boards, colored bars, etc.), different backgrounds (grey, white, black, with grids, etc.), and using different methodologies. For example, Claessen et al. (1995) studied the different expanding behavior of red and the contracting behavior of blue in a puzzle task, where the subjects had to perceive the fit between differently sized pegs and a hole. These studies highlighted the role of the various aspects of color in inducing the dimensional effect (brightness, see Payne, 1964; hue, brightness and saturation, see Tedford et al., 1977; hue and brightness, see Claessen et al., 1995; lightness/darkness, see Gundlach \& Macoubrey, 1931). Caution is therefore necessary in drawing conclusions on the color dimensions responsible for the phenomenon and further research in the field is desirable.

A test of the presence of color dimensions that affect metric size perception in redtail splitfin fish in a human-like fashion might shed further light on other aspects of perceptual organization depending on context or other Gestalt factors in fish. Ingle (1984) showed that goldfish (Carassius auratus), like humans, are able to identify colors very accurately within the context of the background array, rather than by the spectral composition of light reflected by the object. Also in this case, when stimuli differ in specific spectral compositions, color perception in goldfish seems to obey the Gestalt principle of simplicity or economy. We can assume, then, that goldfish are sensitive to "colors," and do not respond to the simple spectral compositions. This occurred even when the spectral composition of the illuminant was radically altered in a generalization test (Ingle, 1984), confirming that the information motivating fish behavior is derived from second order stimuli (i.e., from spatial-temporal relations among first order stimuli through local stimulations). In other words, fish behavior may be also governed by color appearances (Katz, 1935; see also the definition of "perceived color" in the International Lighting Vocabulary). The explanation of the results obtained by Ingle (1984) was given in terms of the retinex theory (Land, 1959; Land \& McCann, 1971). However, it should be noted that even though teleost fish do not have a recognizable laminated neocortex, their telencephalon comprises wide pallial structures homologous to the mammalian neocortex (Jarvis et al., 2005).

Among fish species, color vision has been especially well investigated in goldfish. The number of discriminable spectral colors in goldfish is at least as high as that in human color vision. The ability of goldfish to discriminate spectral colors records the highest values at $400 \mathrm{~nm}, 500 \mathrm{~nm}$ and $610 \mathrm{~nm}$ (Fratzer, Dörr, \& Neumeyer, 1994; Neumeyer, 1986), and it increases with simultaneous color contrast and color constancy for colored papers, with color loci f inside the color tetrahedron $(U V+L M S)$. Goldfish group together spectral color in larger units (Goldman, Lanson, \& Rivera, 1991), showing a kind of categorization analogous to a human color categorization (Zerbolio, 1985), although this is modulated by training wavelength and discrimination ability (Kitschmann \& Neumeyer, 2005). Kitschmann and Neumeyer (2005), however, observed that the influence of training conditions did not necessarily mean that color categories analogous to those represented by color names "red," "green," "yellow," or "blue" in 
human perception do not exist in goldfish. Moreover, the literature suggests that goldfish and other species of the same order (Cyprinodontiformes) have tetrachromatic vision (Kitschmann \& Neumeyer, 2005; Neumeyer, 1985, 1992) due to the use of an ultra-violet-sensitive photoreceptor as the fourth cone type, as observed also in many other vertebrates (Bowmaker, Thorpe, \& Douglas, 1991; Hawryshyn \& Beauchamp, 1985; Neumeyer, 1985, 1986, 1992).

Although color vision has not been directly investigated in our model species - the redtail splitfin (Xenotoca eiseni, family Goodeidae) - it also belongs to the order of Cyprinodontiformes and lives in ecological conditions similar to those of the other species in which tetrachromatic vision has been demonstrated (i.e., sweet, clear and shallow water, with pebbles and aquatic plants). The appearance of quadrichromia in shallow water fish may be due to the optical properties of the aquatic environment, which require at least four classes of cones to achieve an accurate perception of the chromatic properties of objects that equals the one that land animals can achieve with just three classes (Sabbah, Zhu, Hornsby, Kamermans, \& Hawryshyn, 2013).

Color perception is of particular adaptive importance for redtail splitfins because this species exhibits a notable sexual dymorphism in coloration, with males much more colorful than females. Notably, a study conducted on a species of the Goodeidae family (Amarillo, Girardinichthys multiradiatus) phylogenetically close to Xenotoca eiseni demonstrated tetrachromatic color perception and its involvement in females' preferences for male coloration (Garcia \& de Perera, 2002).

Overall, this supports the idea that the Xenotoca eiseni species also has tetrachromatic vision, like other sweet water fish of the super order Atherinomorphs, cyprinodontiformes order (Meyer, Wischnath, $\&$ Foerster, 1985). For the quadricromia in various species of the order Cyprinodontimformes similar evidence exists (see Archer, Endler, Lythgoe, \& Partridge, 1987; Endeman, Klaassen, \& Kamermans, 2013; Neumeyer, 1992), and this is also true for tetrachromatic color vision in goldfish (see Fratzer et al., 1994; Neumeyer, 1992; Palacios, Varela, Srivastava, \& Goldsmith, 1998).

To a certain extent, studies on teleost receptors and their spectral sensitivity can reveal the presence of similarities and differences in the physiological properties of human and fish visual systems. However, these results cannot inform us as to the corresponding color phenomenology as perceived by fish and other animals (for example whether fish perceive a "yellow" without any nuances of "green" or "red"). In the case of UV sensitive animals, one assumes that they see something similar to our "violet," but there is no way to prove this except to test their responsive behavior.

In the research reported here, we tested whether Xenotoca eiseni is sensitive to the so-called chromatic expansion-contraction effects (i.e., dimension illusions due to color). We used isoluminant hues, which excluded the role of brightness in causing the phenomenon: any differences in the behavior of the fish should thus depend on specific attributes of the figure hue. The fish were tested in three different conditions, with three color pairs corresponding to the human yellow-blue, red-green, and redblue. The three color pairs were selected for the following reasons. Double color opponent cells have been found in goldfish at the level of bipolar and ganglion cells (Daw, 1967, 1968; Kaneko \& Tachibana, 1981), and the yellow-blue and red-green pairs have been tested in goldfish as to color contrast (Dörr \& Neumeyer, 1997). As noted, in humans yellow-blue and red-green have been shown to cause effects of enlargement/shrinking (Tedford et al., 1977). As regards red and blue, although not opponent, these colors have been shown to have dimensional effects similar to those of the yellow-blue pair (see Claessen et al., 1995; Yoo \& Smith-Jackson, 2010). In particular, our research hypothesis was that the dimensional phenomenon, if confirmed in fish, would be due to what in human color vision are the psychological/connotative properties of hues rather than merely to the opponence of colors. We therefore tested the influence of these dimensions on two opponent color pairs (yellow-blue; red-green) and a control pair (red and blue). In light of the literature on the dimensional phenomenon in humans, our expectation was that, in the yellow-blue condition, the yellow rectangle would be perceived as larger than the blue one, and that the red rectangle would be perceived as the larger one in both the red-green and red-blue conditions. 


\section{Method}

The training and testing procedures and the experimental apparatus employed in the research corresponded to an established protocol employed in our laboratory, whose standardized description has been excerpted from our previous works (Sovrano et al., 2015, 2016).

\section{Subjects}

Subjects were 10 male mature fish (ranging from 3 to $5 \mathrm{~cm}$ in length) of the species Xenotoca eiseni from a stock maintained in our laboratory within vegetation-rich (Ceratophilllum sp.) large tanks $(55-120 \mathrm{~L})$ provided with artificial illumination, $14 \mathrm{hr}$ per day. Water temperature was maintained at 24 $\pm 2^{\circ} \mathrm{C}$. The fish were fed daily with dry fish food (Sera GVG-Mix $\left.{ }^{\circledR}\right)$. Only males participated in the experiment because of their higher motivation to perform the task in order to rejoin female conspecifics. We decided to use Xenotoca e. as an animal model for comparative reasons, because this species and the procedures we used were also employed in all of our previous studies investigating the perception of visual illusions and of hierarchical configurations (Sovrano \& Bisazza 2008, 2009; Sovrano et al., 2015, 2016; Truppa et al., 2010).

\section{Apparatus}

The apparatus was located in a darkened room and was identical to the apparatus (Figure 1) used in our previous studies (Sovrano et al., 2015, 2016). It consisted of a square tank (15 cm long, $15 \mathrm{~cm}$ wide and $15 \mathrm{~cm}$ high), with uniform white walls (although this term 'white', as observed by Neumeyer (1992), is somewhat inadequate to refer to the white perceived by fish, also in absence of ultraviolet wavelengths, as in our case of incandescent source) and lit centrally with a $75 \mathrm{~W}$ tungsten light bulb. The testing tank was inserted in a larger tank $(60 \mathrm{~cm} \times 36 \mathrm{~cm} \times 25 \mathrm{~cm})$, creating an external surrounding region with vegetation and food, where the test fish was located together with other two conspecifics (females that were not tested) that provided motivation for social reinstatement.

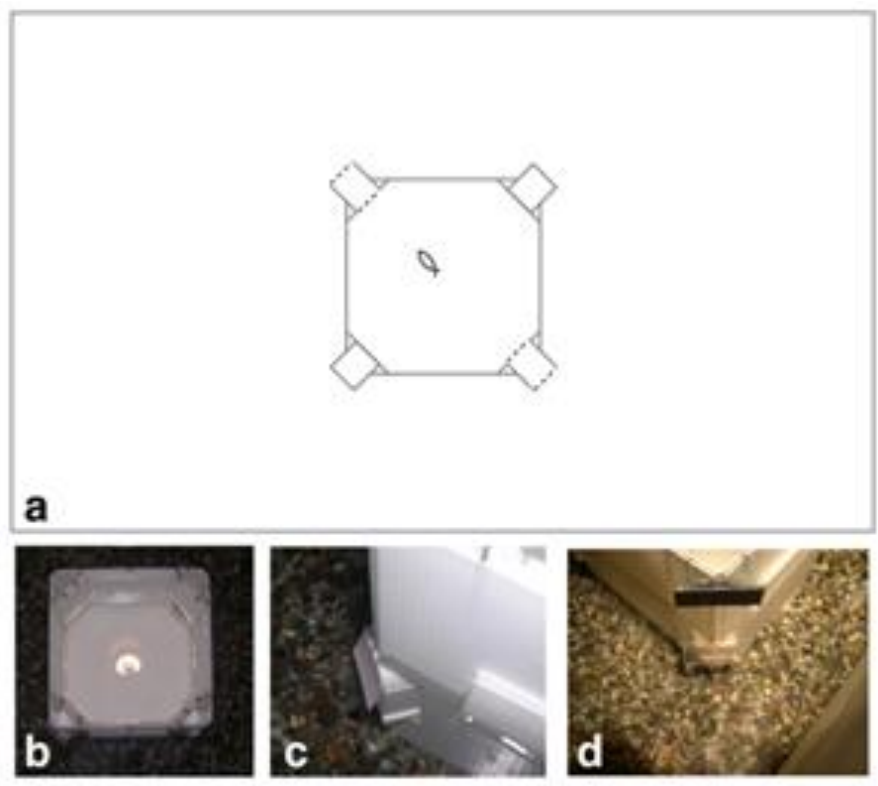

Figure 1. Apparatus used in the experiment: (A) schematic 2D representation of the inner and outer tanks, with an experimental subject portrayed in the center of the inner tank; the dotted lines indicate the tunnels connecting the inner and the outer tank (one of which was blocked to prevent the passage of fish); (B) the inner tank; details of the exit of the open (C) and closed (D) tunnels. 
Inserted in two diagonally opposite corners of the square apparatus was a small tunnel $(2.5 \times 2 \times 3$ $\mathrm{cm}$, located $4.5 \mathrm{~cm}$ from the floor of the tank), of white plastic material, through which the fish could pass to rejoin conspecifics in the surrounding region in the outer tank (see Figure 1). At the end of each tunnel was a door $(2.5 \times 3.5 \mathrm{~cm})$, the upper part of which was a sheet of opaque plastic material, and the lower part a transparent, very flexible sheet of plastic material. The two doors were visually identical, but only one of them could be opened, the other being blocked with an external plastic transparent panel. The fish could thus open the correct door to rejoin conspecifics by pressing on it with the snout; choices of each door were clearly visible in video-recordings, because of characteristic movements of the tail and the most caudal part of the body of the fish, which remained visible outside the tunnel.

The stimuli used for visual discrimination learning and for the illusion-test were located below each door. The stimuli were made of a plastic material designed to resist the aquatic environment. Transparent screens $(9 \times 4 \mathrm{~cm})$ were located $2.3 \mathrm{~cm}$ in front of the stimuli to prevent the fish from getting too close to the stimuli. During training, the stimuli to be discriminated consisted of a large $(1.3 \mathrm{~cm} \mathrm{x} 0.7$ $\mathrm{cm}$ ) and a small $(0.5 \mathrm{~cm} \times 0.4 \mathrm{~cm})$ medium grey rectangle (Figure 2). Half of the subjects were reinforced on the large rectangle, while the other half was reinforced to choose the small one.

When the illusory expansion-contraction effects were tested, the stimuli consisted of two rectangles of identical size $(1.3 \times 0.7 \mathrm{~cm})$, but of different colors (Figure 3$)$. Both test stimuli were of the same size as the larger stimulus used in training, with the consequence that, during the test, the subjects reinforced on the larger rectangle had to choose between two stimuli whose physical size had previously always been reinforced, whereas the subjects reinforced on the smaller training stimulus had to choose between two stimuli whose size had previously never been reinforced.

Across three conditions fish were tested with three different color pairs: i) yellow-blue, with a yellow rectangle generally perceived by humans as larger than blue ones; ii) red-green (opponent colors, with a red rectangle usually perceived by the human observer as expanding, and vice versa the green rectangle); and iii) red-blue (non-opponent, but also known to cause expansion-contraction effects).

The luminance of all training and test stimuli was identical and corresponded to a value of 63.563.8 within the CIELAB color space (measured with a Minolta CS-200 colorimeter). This was done in order to prevent any confound due to luminance differences between training and test stimuli, also in relation to the luminance contrast to the background. Because conditions of isoluminance greatly reduce the chroma of the colored figures and leave only the hue contrast in the background, the experiment mainly tested for the presence and the effect of hue in the perception of size.

The RGB, HSB and CIELAB values used to create the colors are reported in Table 1. Stimuli were printed on a paper whose lightness $\mathrm{L}^{*}$ value in the CIELAB color space was 94.1. 

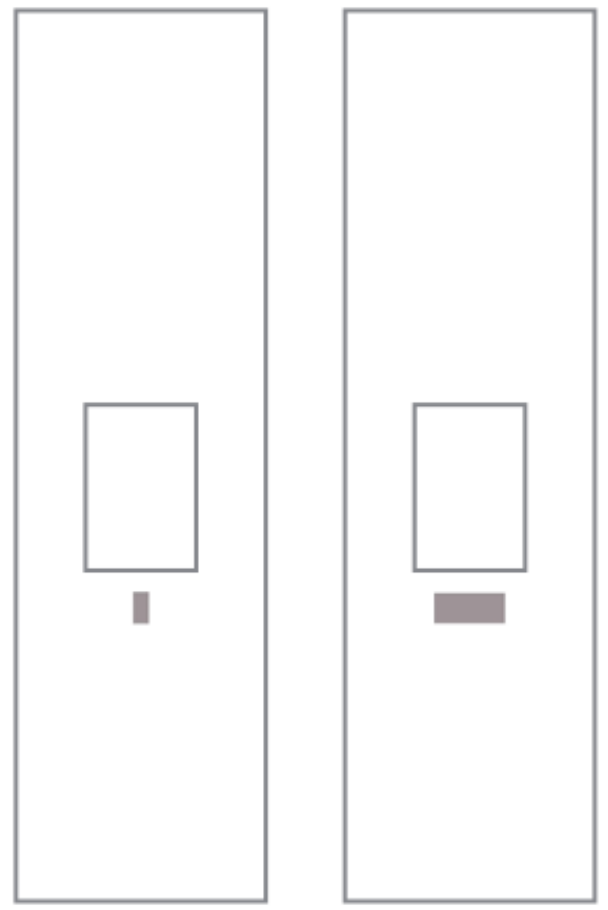

Figure 2. Schematic front view of the tunnel's doors, positioned in two diagonally opposite corners of the squared experimental apparatus: the training-stimuli (a small and a large grey rectangle) are visible below the doors.
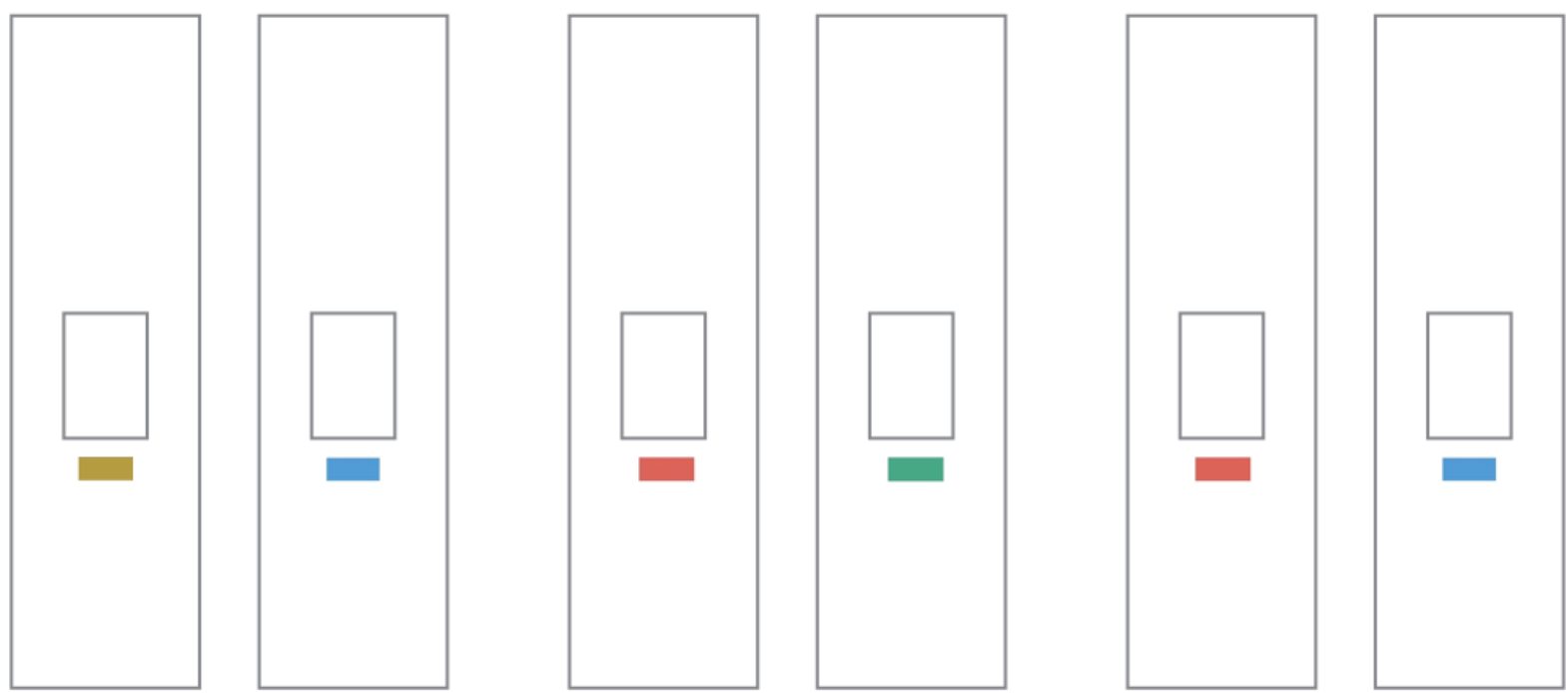

Figure 3. The pairs of isoluminant test-stimuli used in the three different test conditions: yellow-blue, red-green and red-blue equally-sized rectangles. 
Table 1

Color Values

\begin{tabular}{lccccccccc}
\hline & $\mathrm{L}^{*}$ & $\mathrm{a}^{*}$ & $\mathrm{~b} *$ & $\mathrm{R}$ & $\mathrm{G}$ & $\mathrm{B}$ & $\mathrm{H}$ & $\mathrm{S}$ & $\mathrm{B}$ \\
\hline Yellow & 60 & 5 & 57 & 172 & 140 & 33 & 46 & 81 & 68 \\
Red & 62 & 50 & 25 & 235 & 109 & 109 & 0 & 54 & 92 \\
Blue & 55 & -10 & -45 & 38 & 139 & 210 & 204 & 82 & 82 \\
Green & 57 & -50 & 13 & 0 & 158 & 112 & 162 & 100 & 62 \\
Gray & 55 & 6 & 0 & 142 & 128 & 132 & 344 & 10 & 56
\end{tabular}

Note. The first column describes the colors used in the experiment, while the other columns give the 'measures' of those colors in different color systems. Legend: $\mathrm{L}^{*}, \mathrm{a}^{*}, \mathrm{~b}^{*}$ are the dimensions of the CIELAB color space. R, G, B refer primarily to the perceived Red, Green, Blue but usually are the device dependent primaries of monitor colors. H, S, B are the dimensions used in the field of monitors and television $(\mathrm{H}=$ hue; $\mathrm{S}=$ saturation; $\mathrm{B}=$ brightness $)$.

\section{Design and Procedure}

Before testing, for at least one week the fish underwent a "shaping procedure" in their home-tank (30 x $40 \times 20 \mathrm{~cm})$. We used a partition that divided their home-tank into two halves, one ("enriched") with food and vegetation and the other ("unenriched") without any food and vegetation. Two tunnels, with moveable doors identical to those subsequently used during testing, were positioned in the partition so that the fish could move between the two compartments. This made the fish accustomed to the use of the moveable doors before testing. Below the two tunnels, we placed the stimuli, which were then used during the subsequent training. This accustomed the fish to the stimuli and facilitated training. Note that during this phase both moveable doors were associated with the figures later reinforced during training and both allowed the fish to move from one compartment to the other. Selective reinforcement performed by blocking one of the doors was done only during the subsequent training phase, in which the two stimuli of different sizes were presented together.

The experiment proper comprised two parts: training and test. During training, five fish were trained with the large rectangle as the positive reinforced stimulus ("large group"); five fish with the small rectangle as the positive reinforced stimulus ("small group"). The fish were given daily sessions of 10 trials. Training on the discrimination continued until the fish reached the learning criterion $(70 \%$ correct choices in two consecutive sessions).

In each trial, the fish was placed in the inner tank by gently inserting it into a transparent plastic cylinder placed in the center. After $20 \mathrm{~s}$ the cylinder was removed. In each trial the number of choices of the two doors was video-recorded, until the fish was able to exit and rejoin conspecifics (the maximum time was $15 \mathrm{~min}$ ). During training, a correction method (Sutherland \& Mackintosh, 1971) was used: if the fish made a wrong choice, it was allowed to change it until it was able to exit or until the overall time allowed for the trial elapsed. This standard correction method has been used in previous works (e.g., Sovrano \& Bisazza, 2008, 2009; Sovrano et al., 2015, 2016; Truppa et al., 2010), since it is optimal for learning in this task. The inter-trial interval was $7 \mathrm{~min}$ after a correct choice, and $3 \mathrm{~min}$ if fish choices showed mixed responses (incorrect and correct). During the inter-trial interval the fish was allowed to remain in the external surrounding region (reinforcement time). Occasionally, food (the same as that used in the home tank) was given in the outer tank after a correct choice, but not more often than twice in each daily session. After every two trials the test tank was rotated $90^{\circ}$ clockwise, in order to avoid any possible use of extra-tank cues.

After reaching the learning criterion, the fish performed test trials. The test consisted of three single sessions of 10 trials (each trial lasting $2 \mathrm{~min}$ ), one session for each of the three test conditions (yellow-blue, red-green, and red-blue), with each test condition presented in random order to different animals. During the test both doors were closed. If the fish did not make a choice in the 2 min allotted for each trial, they were left in the test tank up to a maximum of $15 \mathrm{~min}$, or until they produced at least one 
choice. This is the standard procedure employed for this task in previous studies (Sovrano \& Bisazza, 2008, 2009; Sovrano et al., 2015, 2016; Truppa et al., 2010), which keeps the fish motivated to make a choice in order to avoid remaining in the central tank for long periods. A minimum of two trials, with the same stimuli as those used during training, were inter-mixed every two-three test-trials, to reinstate the motivation of the fish (these trials were, of course, discarded in the test data analyses). In order to proceed to the next test trials, fish had to perform correctly in both the trials with familiar stimuli. If this was not the case, training trials were repeated until the fish performed correctly two times consecutively.

The total numbers of choices made of each of the two tunnels (immediately above the two rectangle stimuli) during the 2 min of test were recorded for each animal. From these data, we computed the percentage of overall choices for the color hypothesized to cause expansion effect (i.e., for the "perceptually larger" rectangle as seen by human eyes). We expected that the fish that were reinforced on the large rectangle during training and those that were reinforced on the small rectangle during training would differ in their levels of preference for the "perceptually larger" rectangle. In fact, if fish were influenced by the chromatic expansion-contraction effects known in humans, the level of choice for this rectangle (e.g., the yellow one) should have been higher in fish rewarded during the training on the larger grey rectangle than on those rewarded on the smaller rectangle. Moreover, we also wanted to verify whether the chromatic expansion-contraction effects would be equally pronounced in the three colored test conditions or whether they would differ depending on the color pair used.

In a preliminary analysis, we verified that the assumption of normality was met (KolmogorovSmirnov yellow-blue test, $D(10)=0.167, p \geq 0.200$; red-green test, $D(10)=0.322, p \geq 0.2$, red-blue test, $D(10)=0.193, p \geq 0.200$; Shapiro Wilk yellow-blue test $W(10)=0.927, p=0.422$; red-green test, $W(10)$ $=0.899, p \geq 0.212$, red-blue test, $W(10)=0.926, p \geq 0.412$ ). The data were then submitted to an analysis of variance with Test condition (yellow-blue, red-green, and red-blue) as a within-subjects factor and the Reinforced training stimulus (large rectangle vs. small rectangle) used during training as a betweensubjects factor. Differences between the two reinforcement groups were then assessed by two-tailed independent samples $t$-test. Significant departures from random choice $(50 \%)$ were estimated by onesample two-tailed $t$-tests.

\section{Results}

On average fish required 32 trials to complete the training $(\mathrm{SEM}=2.49)$. There was no difference in the number of trials needed to reach the learning criterion between the two groups rewarded with the large or the small grey rectangle (large rectangle: $M \pm \mathrm{SEM}=34 \pm 4.06$; small rectangle: $M \pm \mathrm{SEM}=30$ \pm 3.16 ; 95\% CI $[-7.758,15.758]), t(8)=0.784, p=0.455, d=0.496$. The results of the tests are shown in Figure 4, separately for each condition. Data were analyzed as percentage of choices for the "perceptually larger" color.

The analysis of variance with Test condition (yellow-blue, red-green and red-blue) as a withinsubjects factor and the Reinforced training rectangle (large rectangle vs. small rectangle) as betweensubjects factor revealed a significant interaction between Test condition and Reinforced training rectangle, $F(2,16)=3.675, p=0.049, \eta^{2}=0.315$. There were no other statistically significant effects (Test condition: $F(2,16)=2.532 p=0.111, \eta^{2}=0.24$, Reinforced training rectangle $F(1,8)=1.691, p=0.230$, $\left.\eta^{2}=0.174\right)$. This revealed that the three test conditions differently affected the responses of fish trained on the large and the small rectangle, indicating that the three different color combinations did not cause identical contraction-expansion effects.

Thus, in further analyses we looked at each test condition separately to verify whether and in which conditions we could find a difference between the groups of fish reinforced on the large vs. small training rectangle. In the yellow-blue condition, we found a statistically significant difference between the two reinforcement groups, $t(8)=2.665, p=0.029, d=1.696,95 \%$ CI $[28.14,38.94]$. As expected, the percentage of choice for the yellow rectangle was higher in fish reinforced on the larger grey rectangle than in fish reinforced on the smaller grey rectangle (large group, $M \pm \mathrm{SEM}=67.3 \% \pm 5.85 \%$, small 
group, $M \pm \mathrm{SEM}=46.42 \% \pm 5.2 \%$ ). This indicates that in the yellow-blue condition, at least, fish perceived the chromatic expansion-contraction effect in a similar way to humans, treating the yellow rectangle as larger than the blue rectangle.

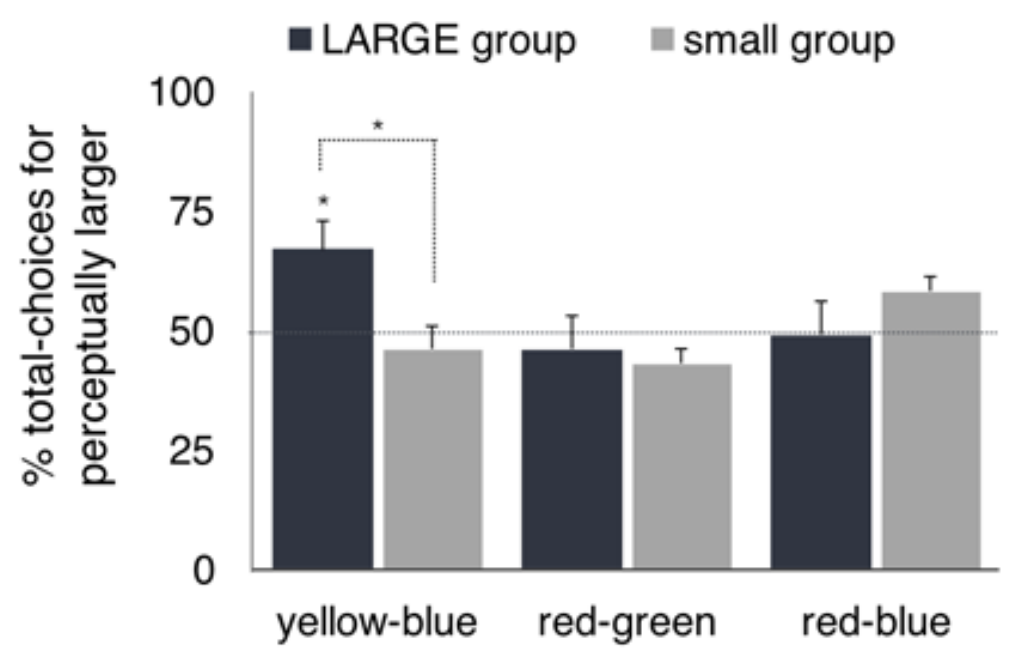

Figure 4. Percentage of total choices for the perceptually larger color for the three test conditions: yellow-blue, red-green, and red-blue. Darker bars represent the performance of fish reinforced on the smaller rectangles; lighter bars represent fish reinforced on the larger ones (group means with SEM are shown). Asterisks $(p<0.05)$ indicate significant differences between the reinforcement groups and significant departures from chance level (represented by the dotted grey line).

Moreover, for subjects reinforced on the large grey rectangle at training the amount of choice for the yellow stimulus was significantly more than what would be expected by chance level (average preference score of $67.3 \%), t(4)=2.954, p=0.042, d=1.321,95 \%$ CI $[1.04,33.55]$. An opposite trend was apparent for the subjects reinforced on the small training rectangle (average preference score of $46.42 \%)$. However, this trend did not approach statistical significance, $t(4)=-0.688, p=0.529, d=0.307$, $95 \%$ CI $[-18.04,10.87]$. The expansion effect thus appeared to be stronger in the group reinforced on the larger rectangle. This may have been due to the fact that both test rectangles, the yellow and the blue one, had the same size as the familiar reinforced training rectangle. Thus, fish of the "large group" that were attracted by the yellow rectangle due to the illusory expansion effect faced a rectangle whose physical size also perfectly corresponded to that of the reinforced training rectangle, possibly strengthening the preference. By contrast, fish of the "small group," that were attracted to the blue rectangle due to the contraction effect, may have been misled by the fact that its actual physical size was that of the nonreinforced training rectangle. However, extreme caution is necessary when deriving interpretations from the lack of significant effects, because it is impossible to rule out lack of statistical power as an alternative explanation. To partially circumvent this problem, we additionally applied a strategy of analysis different with respect to the standard approach used in previous studies. In order to do so, we converted the percentage of choice for the perceptually larger rectangle (yellow) into percentage of choice for the rectangle whose perceived size should better match the reinforced training rectangle of each group. Thus, we computed the percentage of total choice for the yellow rectangle in the "large group" and the percentage of choice for the blue rectangle in the "small group," obtaining for both groups a measure of their choice in the direction expected on assuming a human-like perception of the color effects. The subjects of the two training groups did not differ from each other in these new variables (total choices for the expected rectangle: $t(8)=1.732, p=0.122, d=1.103$ large group, $M \pm \mathrm{SEM}=67.3 \% \pm 5.85 \%$, small group, $M \pm \mathrm{SEM}=53.6 \% \pm 5.31 \%, 95 \% \mathrm{CI}[-45.69,31.93]$. We were thus able to collapse the data of the two groups and compare the performance of the combined sample to chance level. The results revealed that the overall performance of the experimental sample was significantly above chance level, $t(9)=$ 
2.390, $p=0.041, d=0.756(M \pm \mathrm{SEM}=60.45 \% \pm 4.37 \%, 95 \%$ CI $[0.56,20.34])$. We can thus conclude that, overall, fish tested in the yellow-blue condition showed a significant preference for the choice that would be expected on the basis of a human-like perception of the chromatic expansion-contraction effects.

By contrast, no significant differences between the two reinforcement groups were found for either of the other two color conditions, suggesting that the expansion-contraction effects do not extend to these two additional color pairings - red-green: $t(8)=0.423, p=0.684, d=0.274$ (large group, $M \pm$ SEM $=46.4 \% \pm 6.84 \%$, small group, $M \pm \mathrm{SEM}=43.28 \% \pm 2.76 \%, 95 \%$ CI $[-13.91,20.15]$ ); in the red-blue condition, the Levene's test revealed violations of homoscedasticity, thus $d f$ and $p$ values were appropriately corrected: $\mathrm{t}(5.208)=-1.287, p=0.252, d=0.822$ (large group, $M \pm \mathrm{SEM}=49.44 \% \pm$ $6.69 \%$, small group, $M \pm \mathrm{SEM}=58.7 \% \pm 2.63 \%, 95 \% \mathrm{CI}[-27.54,9.02])$.

These results show that the phenomenon does not necessarily have to be explained according to the color opponence because the red-green pair, although it was an opponent pair, did not show any positive result.

\section{Discussion}

As is well known, the connotative or psychological dimensions of color are perceivable by humans, affecting the perceived size of visual objects (Claessen et al., 1995; Cleveland \& McGill, 1983; Gentilucci et al., 2001; Gundlach \& Macoubrey, 1931; Payne, 1964; Tedford et al., 1977; Wallis, 1935; Warden \& Flynn, 1926). The aim of our research was to verify whether male redtail splitfins have, like humans, the tendency to be affected by color dimensions such as expanding/contracting in visual space. The color system of teleost fish, such as goldfish, has been investigated as to their sensitivity to color contrast and color constancy (Daw, 1984), but not in relation to color as a cue responsible for size expansion. Our experiment tested whether identical rectangles of different isoluminant hues, presented in pairs (yellow and blue, red and green, red and blue), were perceived by fish as being of different size (i.e., appearing as smaller or larger than their metric dimensions). Our hypothesis was that, in fish, the perceived size of the rectangles would be affected by the different hues (or better, by the specific connotative dimensions of the hues) responsible for a similar effect in humans. However, because we kept the same conditions of isoluminance for all the stimuli, we instead focused on the role of hue, rather than brightness (Gundlach \& Macoubrey, 1931), in size perception.

During the training and the test phases the rectangles were positioned in the same places, and were hence perceived at the same average distance, indicating that the behavior of the fish was not determined by differences in the perceived distance, but in the perceived size. Our results show that, at least in one of the three conditions (with the yellow and blue pair), the fish perceived and behaved as if the two rectangles were of different sizes. No significant effect, instead, was registered with the other two pairs, red and green and red and blue. This indicates that the males of this species of fish perceive at least some expansion-contract color effects similar to those experienced by human observers (females were not tested in the present study; thus we cannot assume that the effect is present in both genders).

Although the phenomenological terms of color (such as "yellow," "green," "red," and "blue") can be correctly ascribed to human perception only, the behavioral significance of the results obtained with fish for the yellow-blue condition confirms the strength of this pair in the perceived dimensions, which determined the fish choices (in humans yellow is generally the color producing the dimensional effect to the greatest extent). The strength of the yellow and blue pair, in comparison with the red and green pair, cannot be explained as being due to the greater difference between the two hues, since also red and green are equally distant hues.

The difference between the two axes (yellow and blue and red and green) in eliciting the sizeillusion is interesting because of their phylogenetic history within the mammalian lineage. When higherlevel (trichromatic) color vision appeared in mammals, the physiological substrate for the perception of the red-green axis probably emerged from a division of the mechanisms for the perception of the other axis. This happened through differentiation of a photopigment sensitive to the medium waves in two 
different photopigments (Mollon, 1989). In humans, these two photopigments are sensitive to wavelengths that are not very different from each other (currently named medium and long waves). The small difference in wavelength is successively amplified by the nervous system. In this regard, it is worth noting that the diction "R-G" (Red-Green) is often considered equivalent to "M-L" (Medium-Long) wavelength, which are radiation, and therefore are not colored, although yellow, and not red, is perceived by humans when the eye is stimulated by wavelengths around $572 \mathrm{~nm}$, which is the peak of sensitivity of the L cone (Pridmore, 2013). However, it should be noted that color vision's appearance in mammals could be quite peculiar. The original development of the major visual pigment families perhaps occurred early in vertebrate evolution, before the separation of the different vertebrate classes (350 million years ago). The alleged vertebrate ancestors are hypothesized to have first evolved two classes of photoreceptors (LWS and SWS) through a first gene duplication, creating the basis for the dichromatic color vision shared by most vertebrates. Two further gene duplications within the SWS opsin group led to three opsin classes (peaking at about $360-450,440-470$ and $470-520 \mathrm{~nm}$ ). Finally, a fourth gene duplication within the MWS opsins led to the rod opsins $(500 \mathrm{~nm})$. The teleost fish species that show tetrachromatic vision probably inherited this trait from the alleged common vertebrate ancestor. By contrast, the supposed mammalian ancestors could have transitioned to dichromatic vision as an adaptation to nocturnal life, and then (in the case of some primates) they could have adopted a trichromatic color vision when shifting back to diurnal life (Bowmaker, 1998). Thus, the arguments developed for humans could not be directly applied to the same phenomena when they are observed in fish species.

It should also be noted that, in our model species, the spectral sensitivity of the three non-UV cone classes is likely to differ from that of the corresponding photoreceptor classes of humans. Even though photoreceptor spectral sensitivity has never been measured in Xenotoca eiseni, data from the guppy Poecilia reticulata reveal a spectral sensitivity profile different from that observed in humans. The main difference between the two species seems to be in the photoreceptor of intermediate sensitivity, whose peak in guppies is at around $465 \mathrm{~nm}$ compared to the $530 \mathrm{~nm}$ reported in primates (Archer et al., 1987; Baylor, Nunn, \& Schnapf, 1987; Schnapf, Kraft, Nunn, \& Baylor, 1988).

Further studies might capitalize on the current work and investigate the role of luminance further. For example, one might produce a series of stimuli in which each has the luminance related to the natural lightness (Spillmann, 1985) of the single hues, but keeping the series at different levels of luminance (one low, one high), so that the higher luminance of a series is lower than the lower luminance of the successive one. In this way, one could compare colors of the same hue with different luminances. Another factor to be tested, besides brightness and hue, is the role of saturation in producing the size expansion/contraction effect.

From a more general viewpoint, our results show once again that the visually guided behavioral responses of redtail splitfin fish are very similar to those of humans, confirming that remarkable similarities exist in the visual system between fish and other vertebrates. These remarkable similarities may indicate an analogous organization, in distant vertebrates, of the neural circuitry involved in these perceptual effects. It also suggests that the various organisms, independently of the difference among species, have adapted and respond to the environment in similar manners. These comparative studies also shed light on the phenomena of vision with regard to their possible evolutionary implications. In fact, the overall evidence suggests that the perceptual processes subtending the influence of contextual factors are shared by mammals and fish. Fish are particularly interesting for the analysis of perceptual phenomena, both because they are amenable to traditional training procedures and because their abilities can be investigated through naturalistic incidental learning tasks as in our study. Further studies, however, might extend the research on chromatic expansion/contraction effects to diurnal birds, which live in different ecological conditions but have the potential for tetrachromatic color vision (Bowmaker, 1998), to verify the actual perceived dimensions of objects in that specific visual environment. 


\section{Acknowledgements}

We wish to thank Matteo Kettmaier for his help with the experiments and Francesco Cerri for the maintenance of the aquariums.

Conflicts of Interest: The authors declare no conflict of interest.

Ethical Statements: The present research was carried out in the Animal Cognition and Neuroscience Laboratory (A.C.N. Lab.) of the CIMeC (Center for Mind/Brain Sciences) at the University of Trento (Italy). Animal husbandry and experimental procedures complied with European Legislation for the Protection of Animals used for Scientific Purposes (Directive 2010/63/EU) and the Italian law. Experimental procedures have been authorized by the University of Trento's Ethics Committee for the Experiments on Living Organisms and the Italian Ministry of Health (authorization protocol: 2012021A). The number of animals employed in the experiments is closely consistent with the alternative method of "Reduction," which allows us to use only the minimum number of animals useful to draw statistically valid conclusions.

\section{References}

Albers, J. (1975). Interaction of colour (Revised ed.). New Haven, CT \& London: Yale University.

Agrillo, C., Miletto Petrazzini, M. E., \& Bisazza, A. (2016). Brightness illusion in the guppy (Poecilia reticulata). Journal of Comparative Psychology, 130, 55-61.

Agrillo, C., Miletto Petrazzini, M. E., \& Dadda, M. (2013). Illusory patterns are fishy for fish, too. Frontiers in Neural Circuits, 7, 137.

Archer, S. N., Endler, J. A., Lythgoe, J. N., \& Partridge, J. C. (1987). Visual pigment polymorphism in the guppy Poecilia reticulata. Vision Research, 27, 1243-1252.

Baylor, D. A., Nunn, B. J., \& Schnapf, J. L. (1987). Spectral sensitivity of cones of the monkey Macaca fascicularis. Journal of Physiology, 390, 145-160.

Bazzeo, A., Zanforlin, M. (1984). Apparent shrinkage and expansion of striped surfaces. Paper presented at the 28 January 1984 meeting of the Accademia Patavina di Scienze, Lettere ed Arti.

Bazzeo, A., \& Zanuttini, L. (1981). The perceived size of textured surfaces. The Italian Journal of Psychology, 8, $103-110$.

Beauchamp, R. D. (1978). Color vision in goldfish: A comparison between psychophysical and neurophysiological findings. In J. C. Armington, J. Krauskopf, \& B Wooten (Eds.), Visual psychophysics and physiology: A volume dedicated to Lorrin Riggs (pp. 63-71). New York, NY: Academic Press.

Bowmaker, J. K. (1998). Evolution of colour vision in vertebrates. Eye, 12, 541-547.

Bowmaker, J. K., Thorpe, A., \& Douglas, R. H. (1991). Ultraviolet sensitive cones in goldfish. Vision Research, 31, 349-352.

Burkamp, W. (1923). Versuche über das Farbenwiedererkennen der Fische. Zeitschrift für Sinnesphysiologie, 55, $133-170$.

Callander, S., Jennions, M. D., \& Backwell, P. R. Y. (2011). Female choice over short and long distances: Neighbour effects. Behavioral Ecology and Sociobiology, 65, 2071-2078.

Claessen. J. P., Overbeeke, C. J., \& Smets, G. J. F. (1995). Puzzling colours. Color Research and Application, 20, 388-396.

Cleveland, W. S., \& McGill, R. (1983). A color caused optical illusion on a statistical graph. The American Statistician, 37, 101-105.

Coren, S., \& Gyrus, J. S. (1978). Seeing is deceiving: The psychology of visual illusions. New York, NY: Lawrence Erlbaum Associates.

Da Pos, O., \& Valenti, V. (2007, July). Warm and cold colours. Paper presented at the Midterm Colour Meeting of the International Colour Association: Colour Science for Industry, Hangzhou, China.

Daw, N. W. (1967). Goldfish retina: Organization for simultaneous contrast. Science, 158, 942-944.

Daw, N. W. (1968). Colour-coded ganglion cells in goldfish retina: Extension of their receptive fields by means of the new stimuli. Journal of Physiology, 197, 567-592. 
Daw, N. W. (1984). The psychology and physiology of colour vision. Trends in Neuroscience, 7, 330-335.

Dörr, S., \& Neumeyer, C. (1996). The goldfish - a colour constant animal. Perception, 25, 243-250.

Dörr, S., \& Neumeyer, C. (1997). Simultaneous contrast in goldfish - a quantitative study. Vision Research, 37, $1581-1593$.

Endeman, D., Klaassen, L. J., \& Kamermans, M. (2013). Action spectra of zebrafish cone photoreceptors. PLoS ONE, 8, e68540.

Fratzer, C., Dörr, S., \& Neumeyer, C. (1994). Wavelength discrimination of the goldfish in the ultraviolet spectral range. Vision Research, 34, 1515-1520.

Fuss, T., Bleckmann, H., \& Schluessell, V. (2013). The brain creates illusions not just for us: Sharks (Chiloscyllium griseum) can "see the magic" as well. Frontiers in Neural Circuits, 8, 24.

Garcia, M. C., \& de Perera, T. (2002). Ultraviolet-based female preferences in a viviparous fish. Behavioral Ecology and Sociobiology, 52, 1-6.

Gentilucci, M., Benuzzi, F., Bertolani, L., \& Gangitano, M. (2001). Influence of stimulus color on the control of reaching-grasping movements. Experimental Brain Research, 137, 36-44.

Goldman, M., Lanson, R., \& Rivera, G. (1991). Wavelength categorization by goldfish (Carassius auratus). International Journal of Comparative Psychology, 4, 195-209.

Gori, S., Agrillo, C., Dadda, M., \& Bisazza, A. (2014). Do fish perceive illusory motion? Scientific Reports, $4,6443$.

Gregory, R. L. (1966). Eye and brain. New York, NY: McGraw Hill.

Gundlach, C., \& Macoubrey, C. (1931). The effect of color on apparent size. American Journal of Psychology, 43, 109-111.

Hård, A, \& Sivik, L. (2001). A theory of colors in combination: A descriptive model related to the NCS colour-order system. Colour Research and Application, 26, 4-28.

Hawryshyn, C. W., \& Beauchamp, R. (1985). Ultraviolet photosensitivity in goldfish: An independent UV retinal mechanism. Vision Research, 25, 11-20.

Henrich, J. (2008). A cultural species. In M. Brown (Ed.) Explaining culture scientifically (pp. 184-210). Seattle, WA: University of Washington Press.

Henrich, J., Heine, S. J., \& Norenzayan, A. (2010). The weirdest people in the world? Behavioural Brain Research, $33,61-135$.

Herter, K. (1950). Über simultanen Farbkontrast bei Fischen. Zeitschrift für Vergleichende Physiologie, 60, 283300.

Ingle, D. I. (1984). The goldfish as a Retinex animal. Science, 227, 651-653.

Itten, J. (1961). Kunst der Farbe. Ravensburg: Otto Maier Verlag.

Jarvis, E. D., Güntürkün, O., Bruce, L., Csillag, A., Karten, H., Kuenzel, W., \& Butler, A. B. (2005). Avian brains and a new understanding of vertebrate brain evolution. Nature Reviews Neuroscience, 6, 151-159.

Kandinsky, W. (2001) Concerning the spiritual in art. New York, NY: MFA Publications.

Kaneko, A., \& Tachibana, M. (1981). Retinal bipolar cells with double colour-opponent receptive fields. Nature, 293, 220-222.

Kanizsa, G. (1972). Schrumpfung von visuellen Feldern bei amodaler Ergänzung. Studia Psychologica, 14, 208210.

Kanizsa, G., \& Luccio, R. (1978). Espansione di superfici da completamento amodale. Reports from the Institute of Psychology, Trieste: University of Trieste.

Katz, D. (1935). The world of colour. London, UK: Kegan Paul.

Kelley, L. A., \& Kelley, J. L. (2014). Animal visual illusion and confusion: The importance of a perceptual perspective. Behavioral Ecology, 23, 1-14.

Kiesow, F. (1906). Über einige geometrisch-optische Täuschungen. Archiv für die gesamte Psychologie, 6, 289305.

Kitschmann, M., \& Neumeyer, C. (2005). Generalization and categorization of spectral colors in goldfish I. Experiments with one training wavelength. Journal of Comparative Physiology A, 191, 1025-1036.

Klee, P. (1961). Notebooks, vol. 1. The thinking eye. London-New York: Lund Humphries George Wittenborn.

Kumar, S., \& Hedges, S. B. (1998). A molecular timescale for vertebrate evolution. Nature, 392, 917-920.

Land, E. H. (1959). Color vision and the natural image. Part I. Proceedings of the National Academy of Sciences of the United States of America, 45, 115-129.

Land, E. H., \& McCann, J. J. (1971). Lightness and retinex theory. Journal of the Optical Society of America, 61, 111.

Mascalzoni, E., \& Regolin, L. (2011). Animal visual perception. Wiley Interdisciplinary Reviews: Cognitive Science, 2, $106-116$. 
Meyer, M. K., Wischnath, L., \& Foerster, W. (1985). Lebendgebärende Zierfishe: Arten der Welt [Viviparous ornamental fishes: Species of the world]. Melle, Germany: Mergus Verlag.

Mollon, J. (1989). Tho' she kneel'd in that place where they grew... The uses and origins of primate colour vision. Journal of Experimental Biology, 146, 21-38.

Neumeyer, C. J. (1985). An ultra-violet receptor as a fourth receptor type in goldfish color vision. Naturwissenschaften, 72, 162-163.

Neumeyer, C. (1986). Wavelength discrimination in goldfish. Journal of Comparative Phsyiology A, 158, $203-213$.

Neumeyer, C. J. (1992). Tetrachromatic color vision in goldfish: Evidence from color mixture experiments. Journal of Comparative Physiology A, 171, 639-649.

Nieder, A. (2002). Seeing more than meets the eye: Processing of illusory contours in animals. Journal of Comparative Physiology A, 188, 249-260.

Oyama, T. (1960). Japanese studies on the so-called geometrical-optical illusions. Psychologia, 3, 7-20.

Oyama, T., \& Jitsumori, M. (1973). Behavioral study of color mixture in the carp. Vision Research, 13, $2229-2308$.

Palacios, A. G., Varela, F. J., Srivastava, R., \& Goldsmith, T. H. (1998). Spectral sensitivity of cones in the goldfish, Carassius auratus. Vision Research, 38, 2135-2146.

Payne, M. C. J. (1964). Color as independent variable in perceptual research. Psychological Bulletin, 61, $199-208$.

Pridmore, R. W. (2013). Cone photoreceptor sensitivities and unique hue chromatic responses: Correlation and causation imply the physiological basis of unique hues. PLOS ONE, 8, e77134.

Reaney, L. T. (2009). Female preference for male phenotypic traits in a fiddler crab: Do females use absolute or comparative evaluation? Animal Behaviour, 77, 139-143.

Rosa-Salva, O., Rugani, R., Cavazzana, A., Regolin, L., \& Vallortigara, G. (2013). Perception of the Ebbinghaus illusion in four-day-old domestic chicks (Gallus gallus). Animal Cognition, 16, 895-906.

Rosa-Salva, O., Sovrano, V. A., \& Vallortigara, G. (2014). What can fish brains tell us about visual perception? Frontiers in Neural Circuits, 8, 119.

Sabbah, S., Zhu, C., Hornsby, M. A. W., Kamermans, M., \& Hawryshyn, C. W. (2013). Feedback from horizontal cells to cones mediates color induction and may facilitate color constancy in rainbow trout. PLoS ONE, 8, e66216.

Schnapf, J. L., Kraft, T. W., Nunn, B. J., \& Baylor, D. A. (1988). Spectral sensitivity of primate photoreceptors. Visual Neuroscience, 1, 255-261.

Segall, M. H., Campbell, D. T., \& Herskovits, M. J. (1963). Cultural différences in the perception of geometric illusions. Science, 139, 769-771.

Schopenhauer, A. (1994). On vision and colors. Oxford, UK: Berg Publishers, Inc.

Sovrano, V. A., Albertazzi, L., \& Rosa-Salva, O. (2015). The Ebbinghaus illusion in a fish (Xenotoca eiseni). Animal Cognition, 18, 533-542.

Sovrano, V. A., Bisazza, A. (2008). Recognition of partly occluded objects by fish. Animal Cognition, 11, $161-166$.

Sovrano, V. A., \& Bisazza, A. (2009). Perception of subjective contours in fish. Perception, 38, 579-590.

Sovrano, V. A., Da Pos, O., \& Albertazzi, L. (2016). The Müller-Lyer illusion in the teleost fish (Xenotoca eiseni). Animal Cognition, 19, 123-132.

Spillmann, W. (1985, June). The concept of lightness ratio of hues in colour combination theory. Paper presented at the $5^{\text {th }}$ Congress of the International Colour Association. Montecarlo: Mondial Color 85. Paris, France.

Sutherland, N. S. (1961). The methods and findings of experiments on the visual discrimination of shape by animals. Quarterly Journal of Experimental Psychology. Monographs, 1, 1-68.

Sutherland, N. S, \& Mackintosh, N. J. (1971). Mechanisms of animal discrimination learning. New York, NY: Academic Press.

Tedford, W. H, Bergquist, S. L., \& Flynn W. E. (1977). The size-color illusion. Journal of General Psychology, 97, $145-149$.

Truppa, V., Sovrano, V. A., Spinozzi, G., \& Bisazza, A. (2010). Processing of visual hierarchical stimuli by fish (Xenoteca eiseni). Behavioural Brain Research, 207, 51-60.

Vallortigara, G. (2006). The cognitive chicken: Visual and spatial cognition in a non-mammalian brain. In E. A. Wasserman \& T. R. Zentall (Eds.) Comparative cognition: Experimental explorations of animal intelligence (pp. 41-58). Oxford, UK: Oxford University Press.

Vallortigara, G. (2009). Original knowledge and the two cultures. In E. Carafoli, G. A. Danieli, G. O. Longo (Eds.), The two cultures: Shared problems (pp. 125-145). New York, NY: Springer Verlag.

Vallortigara, G. (2012). Core knowledge of object, number, and geometry: A comparative and neural approach. Cognitive Neuropsychology, 29, 213-236. 
Vallortigara, G., Chiandetti, C., Rugani, R., Sovrano, V. A., \& Regolin, L. (2010). Animal cognition. Wiley Interdisciplinary Reviews: Cognitive Science, 1, 882-893.

Wade, N. J. (2005). Perception and illusions, historical perspectives. Dordrecht, Germany: Springer.

Wade, N. J. (2010). Visual illusions. Corsini Encyclopedia of Psychology, 1-2.

Wallis, W. A. (1935). The influence of color on apparent size. Journal of General Psychology, 13, 193-199.

Warden, C. J., \& Flynn, E. L. (1926). The effect of color on apparent size and weight. The American Journal of Psychology, 37, 398-401.

Wyzisk, K. (2005). Experimente zur Form und Größenwahrnehmung beim Goldfish (Carassius auratus) unter Verwendung von Scheinkonturen und Größentäuschungen. Unpublished dissertation, Johannes Gutenberg Universität Mainz, Germany.

Wyzisk, K., \& Neumeyer, C. (2007). Perception of illusory surfaces and contours in goldfish. Visual Neuroscience, 24, 291-298.

Yoo, H. S., \& Smith-Jackson, T. L. (2010). Colour size illusion on liquid crystal displays and design guidelines for bioinformatics tools. Behaviour \& Information Technology, 30, 775-785.

Zerbolio, D. J. J. (1985). Categorical color coding in goldfish. Animal Learning and Behaviour, 13, 269-273. 